\title{
Serum bilirubin is significantly associated with Hba lc in type 2 diabetic subjects
}

\begin{abstract}
Diabetes mellitusis a group of metabolic diseases characterized by increase blood glucose level defects in insulin secretion and insulin action. Bilirubin has anti-inflammatory, antiapoptotic and antioxidant properties. As an antioxidant, it has been shown to suppress the oxidation of lipids and lipoproteins; especially LDL-cholesterol.The present study was conducted to assess the relationship between serum bilirubin levels with insulin resistance and other potential risk factors of diabetes in type 2diabetic subjects. This cross sectional study was conducted on 200 type 2diabetic subjects who attended Amin Hayat Memorial Hospital in Lahore. Patients with age group 27-64years were included in the study. Out of which 90 were males and 110 were females. Different demographic and biochemical parameters as age, BMI,WHR, Blood pressure, personal history and socioeconomic status were recorded while fasting blood glucose and random blood glucose. HbA c c, lipid profile and serum bilirubin levels, serum insulin by chemistry analyzer and ELISA. HOMA IR was calculated.
\end{abstract}

The level of bilirubin showed negative significant association with fasting blood glucose, $\mathrm{HbAIc}$ and cholesterol, LDL-cholesterol and triglycerides while non significant association was observed with BMI $\left(\mathrm{Kg} / \mathrm{m}^{2}\right)$,WHR, B.P

Keywords: diabetes mellitus, bilirubin, lipid metabolism, HbAIc, antioxidant
Volume 5 Issue 6 - 2017

\author{
Tasnim Farasat, Saima Sharif, Farkhanda \\ Manzoor, Shaugfta Naz \\ Department of Zoology, Lahore College for Women University,
} Pakistan

Correspondence: Saima Sharif, Department of Zoology, Lahore College for Women University, Jail Road, Lahore, Pakistan,Tel 03334092232,Email ssharif1978@yahoo.com

Received: October 24, 2017 | Published: December 4, 2017

\section{Introduction}

Diabetes mellitus is a syndrome of disordered metabolism, usually due to a combination of hereditary and environmental causes, resulting in abnormally high blood sugar levels (Hyperglycemia). Type 2diabetes is the more common form and accounts for 90$95 \%$ of all diabetes cases worldwide. Diabetes can promote and perpetuate oxidative stress through multiple mechanisms including hyperglycemia and increased free fatty acids. As oxidative stress has been implicated in the pathogenesis of the microvascular and macrocirculatory complications of diabetes. ${ }^{1}$

Disturbance of lipid metabolism appears to be an early event in the development of type 2 diabetes, potentially preceding the disease by several years. ${ }^{2}$ In addition, the different components of diabetic dyslipidemia are believed to be metabolically linked., ${ }^{3,4}$ The dyslipidemia associated with insulin resistance. Accumulating evidence indicates that lipid and glucose homeostasis is interrelated. Both are affected by bile acid-activated signaling pathways in the liver. ${ }^{6}$ Indeed, bile acids have an established role in dietary lipid absorption and cholesterol metabolism, and are also signaling molecules that affect systemic endocrine functions through multiple signaling pathways. $^{7}$ Agents that modulate bile acids may potentially affect both cholesterol and glucose metabolism, and hence dyslipidemia and hyperglycemia, in patients with type 2diabetes. ${ }^{5,6,8}$

Bilirubin is an endogenous compound that can be toxic. ${ }^{9}$ However, it has recently been recognized that unconjugated bilirubin (UCB) exerts a strong anti-oxidant activity, and that mild hyperbilirubinaemia might have positive health effects. Bilirubin is the ultimate breakdown product of haemoglobin and serves as a diagnostic marker of liver and blood disorders. Bilirubin derives from haem present in haemoglobin and is released during breakdown of senescent erythrocytes, whereas approximately $20 \%$ of the daily production is derived from haem proteins such as the cytochrome P 450 isoenzymes. Bilirubin has been regarded as a powerful endogenous anti-oxidant and antiinflammatory agent..$^{10}$ As an antioxidant, it has been shown to suppress the oxidation of lipids and lipoproteins, especially LDL-cholesterol, ${ }^{11}$ and to be directly related to the total serum antioxidant capacity in humans. ${ }^{12}$ Studies indicate that the lipophilic bilirubin is more effective at protecting lipids from oxidation than the water-soluble antioxidants such as glutathione, which primarily protect proteins from oxidation. ${ }^{13}$

Physiological levels of total bilirubin block the production of various free radicals that might hinder the inhibitory responses of the cell to take up the high glucose..$^{14}$ Furthermore, it is shown to prevent the vascular endothelial activation from the oxidative stress in the vessels. ${ }^{15,16}$ TBili are protective against cardiovascular disease, stroke and peripheral arterial disease. ${ }^{17}$

The liver helps maintain normal blood glucose concentration in the fasting and postprandial state. Loss of insulin effect on the liver leads to glycogenolysis and an increase in hepatic glucose production. Abnormalities of triglyceride storage and lipolysis in insulin-sensitive tissues such as the liver are an early manifestation of conditions characterized by insulin resistance and are detectable earlier than fasting hyperglycemia. The precise genetic, environmental, and metabolic factors are sequence of events that lead to the insulin resistance and thus diabetes mellitus. ${ }^{17}$ The present study was conducted to assess the serum bilirubin and its relationship with insulin resistance and other parameters.

\section{Materials and methods}

The cross sectional study was conducted on 250 type 2 diabetic patients. The blood samples of 250diabetic subjects were collected 
by visiting the Amin Hayat Memorial Hospital, Lahore. The study carried out after the approval by the Ethical Committee of the Board of Directors, Hamza Foundation at Amin Hayat Hospital, Out of 250diabetic subjects, 50patients that were taking medication for hypertension and dyslipidemia were excluded from the study.

The questionnaires were filled to collect the personal information of the patients. Information about their life style, socioeconomic status and history of past illness were also included in questionnaire. The anthropometric characteristics such as BMI and WHR and blood pressure were measured and recorded before the samples were taken.

\section{Sample collection and analysis}

Both fasting and random blood samples of 200 diabetic patients were taken. Blood was drawn by using sterile syringes and collected in vacuum tubes. The serum was separated by centrifuging the sample at 4000rpm for 20 minutes. Serum was separated by using micropipette and collected in eppendorfs and stored at $-20^{\circ} \mathrm{C}$ in refrigerator until analysis. Multiple laboratory analysis was performed at the
Amin Hayat diabetic center. These biochemical tests included both fasting and random blood glucose and $\mathrm{HbA} 1 \mathrm{c}$ test. The lipid profiles which include cholesterol, HDL-cholesterol, LDL-cholesterol, and triglycerides were measured by chemistry analyzer and serum bilirubin level was assayed by using assay kit in Lahore College for Women University labs.

\section{Statistical analysis}

All the statistical analysis was performed by using the SPSS version 13.0 (ILO, Chicago). The descriptive statistics was applied to calculate the mean, standard error. Pearson's correlation was applied to relate the level of bilirubin with other parameters of type 2 diabetic subjects.

\section{Results} $1)$.

The mean + SEM of the study parameters are presented in (Table

Table I The mean \pm SEM values of both demographic and biochemical parameters

\begin{tabular}{|c|c|c|c|}
\hline Parameters & All $(n=200)$ & Male(n=90) & Female $(n=110)$ \\
\hline Age (year) & $48.5 I \pm 0.70$ & $49.24 \pm 1.18$ & $48.05 \pm 0.90$ \\
\hline Height (cm) & $161.05 \pm 0.63$ & $167.25 \pm 0.86$ & $155.72 \pm 0.55$ \\
\hline Weight (Kg) & $71.94 \pm 1.001$ & $73.16 \pm 1.54$ & $70.69 \pm 1.36$ \\
\hline BMI Kg/m² & $27.8 I \pm 0.37$ & $26.14 \pm 0.47$ & $29.14 \pm 0.54$ \\
\hline Waist $(\mathrm{cm})$ & $99.05 \pm 0.89$ & $96.32 \pm 1.29$ & $101.09 \pm 1.25$ \\
\hline Hip (cm) & $97.92 \pm 0.68$ & $94.63 \pm 0.87$ & $100.47 \pm 0.96$ \\
\hline WHR & $1.09 \pm 0.06$ & $1.11 \pm 0.09$ & $1.07 \pm 0.07$ \\
\hline Fasting $\mathrm{mg} / \mathrm{dL}$ & $138.57 \pm 0.49$ & $138.90 \pm 0.79$ & $138.54 \pm 0.64$ \\
\hline Random mg/dL & $289.48 \pm 2.63$ & $290.92 \pm 4.3$ & $288.52 \pm 3.40$ \\
\hline HbAlc \% & $8.54 \pm 0.092$ & $8.7 I \pm 0.13$ & $8.43 \pm 0.13$ \\
\hline Systolic BP mmHg & $131.74 \pm 0.56$ & $131.17 \pm 0.93$ & $132.32 \pm 0.73$ \\
\hline Diastolic BP mmHg & $87.18 \pm 0.54$ & $86.33 \pm 0.86$ & $87.91 \pm 0.76$ \\
\hline Cholesterol mg/dL & $205.59 \pm 3.32$ & $197.09 \pm 4.89$ & $212.40 \pm 4.6$ \\
\hline HDL-Cholesterol mg/dL & $37.77 \pm 0.39$ & $36.17 \pm 0.57$ & $39.03 \pm 0.51$ \\
\hline LDL-Cholesterol mg/dL & $230.85 \pm 1.9$ & $227.05 \pm 2.72$ & $233.57 \pm 2.87$ \\
\hline Triglycerides mg/dL & $229.15 \pm 5.12$ & $229.40 \pm 7.75$ & $228.8 I \pm 6.96$ \\
\hline Bilirubin (mg/dL) & $0.62 \pm 0.02$ & $0.68 \pm 0.03$ & $0.58 \pm 0.02$ \\
\hline Insulin resistance $\mu \mathrm{IU} / \mathrm{ml}$ & $28.1 \pm 2.3$ & $29.25 \pm 1.83$ & $23.32 \pm 5.3$ \\
\hline HOMA IR & 9.61 & 10.03 & 7.98 \\
\hline
\end{tabular}

Significance * level $(\mathrm{p}<0.05)$ 


\section{Correlation analysis}

Correlation of bilirubin with BMI: The level of bilirubin (mg/ $\mathrm{dL})$ showed negative non-significant association with BMI $\left(\mathrm{Kg} / \mathrm{m}^{2}\right)$ $(\mathrm{r}=-0.039 ; \mathrm{p}>0.05)$.

Correlation of bilirubin with WHR: Bilirubin level $(\mathrm{mg} / \mathrm{dL})$ was negatively associated with WHR $(\mathrm{r}=-0.011 ; \mathrm{p}>0.05)$ but this association is non-significant.

Correlation of bilirubin with fasting: The level of bilirubin showed negative significant correlation with the fasting glucose (mg/ dL) $(\mathrm{r}=-0.267 ; \mathrm{p}<0.01)$.

Correlation of bilirubin with systolic blood pressure: Nonsignificant association of bilirubin $(\mathrm{mg} / \mathrm{dL})$ with systolic blood pressure $(\mathrm{mmHg})(\mathrm{r}=-0.006 ; \mathrm{p}>0.05)$ was observed.

Correlation of bilirubin with HbA1c: The level of bilirubin showed significant association with HbA1c $(\%)(r=0.70 ; p<0.05)$.

Correlation of bilirubin with cholesterol: The concentration of bilirubin $(\mathrm{mg} / \mathrm{dL})$ showed negative significant association with cholesterol $(\mathrm{mg} / \mathrm{dL})(\mathrm{r}=-0.446 ; \mathrm{p}<0.01)$ (Figure 1).

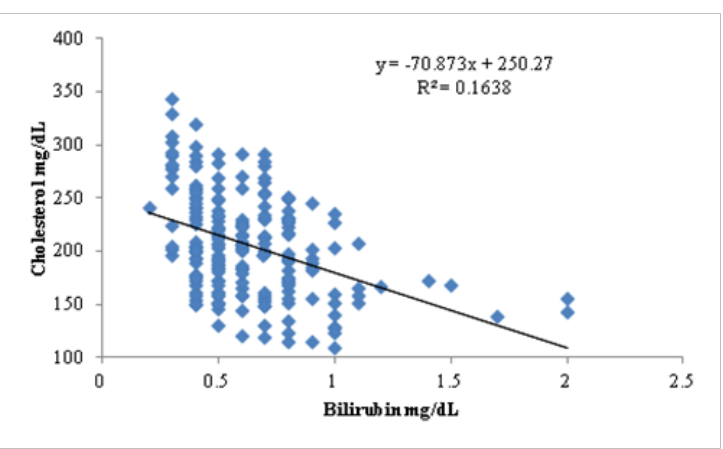

Figure I Correlation between bilirubin level $(\mathrm{mg} / \mathrm{dL})$ and cholesterol $(\mathrm{mg} / \mathrm{dL})$ of the type 2 diabetic subjects.

Correlation of bilirubin with HDL-cholesterol: Bilirubin level $(\mathrm{mg} / \mathrm{dL})$ was not significantly linked with HDL-cholesterol $(\mathrm{mg} / \mathrm{dL})$ $(\mathrm{r}=0.031 ; \mathrm{p}>0.05)$.

Correlation of bilirubin with LDL-cholesterol: The concentration of bilirubin $(\mathrm{mg} / \mathrm{dL})$ form negative significant association with LDLcholesterol $(\mathrm{mg} / \mathrm{dL})(\mathrm{r}=-0.349 ; \mathrm{p}<0.01)$ (Figure 2).

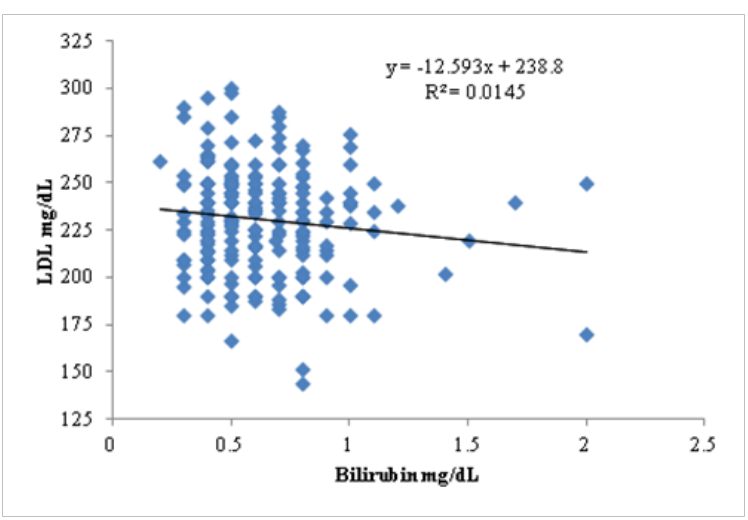

Figure 2 Correlation between bilirubin level $(\mathrm{mg} / \mathrm{dL})$ and LDL-cholesterol $(\mathrm{mg} / \mathrm{dL})$ of the type 2 diabetic subjects.
Correlation of bilirubin with Triglycerides: The level of bilirubin $(\mathrm{mg} / \mathrm{dL})$ showed negative significant association with triglycerides $(\mathrm{mg} / \mathrm{dL})(\mathrm{r}=-0.365 ; \mathrm{p}<0.01)$ (Figure 3$)$.

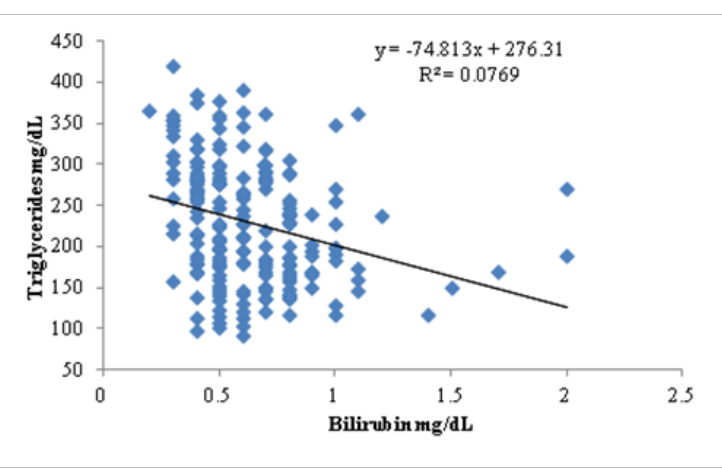

Figure 3 Correlation between level of bilirubin $(\mathrm{mg} / \mathrm{dL})$ and triglycerides $(\mathrm{mg} /$ $\mathrm{dL})$ of the type 2 diabetic subjects.

The results indicate significant inverse association of serum bilirubin with HbA1c, Insulin and IR, total cholesterol, LDL, triglycerides. While significant positive association with HDL cholesterol was observed.

\section{Discussion}

The present study was conducted to demonstrate significant association of bilirubin with other potential risk factors of diabetes. Present study demonstrates non significant association between bilirubin and BMI ( $>0.05)$. Results are inconsistent with the study which revealed negative association between bilirubin concentrations and abdominal obesity. ${ }^{18,19}$ This might be due to low amount of visceral fat and metabolically healthy subjects in our study. Current study revealed negative significant association between bilirubin and fasting glucose $(\mathrm{p}<0.01)$ that is consistent with the study by Lin and his colleagues (2010) who reported negative associations between serum bilirubin concentrations and abnormal glucose level. Studies suggest that an increased expression of heme oxygenase, an enzyme used to break down the hemoglobin into bilirubin, is associated with enhanced insulin sensitivity and glucose metabolism, thus resulting in greater rates of rat model euglycemia. ${ }^{20,21}$ Furthermore, the antioxidant properties of TBili have been postulated to reverse oxidative damage associated with a hyperglycemic state..$^{22}$

Present study demonstrated significant relationship between bilirubin and LDL-cholesterol $(\mathrm{p}<0.01)$. This is inconsistent with the study by kharb. ${ }^{23}$ who reported an inverse relationship between increase in total bilirubin and serum levels of LDL-cholesterol. Study revealed significant relationship between bilirubin $(\mathrm{mg} / \mathrm{dL})$ and triglycerides $(\mathrm{mg} / \mathrm{dL})(\mathrm{p}<0.01)$. This is in favour with the findings of Lalitha $\&$ his colleagues. ${ }^{24}$ who reported increased triglycerides and cholesterol levels in the individuals suffering from diabetes. Current study demonstrates non significant relationship between bilirubin (mg/ $\mathrm{dL})$ and HDL-cholesterol $(\mathrm{p}>0.05)$. This is in favour with study by Suryawanshi et al. ${ }^{25}$ who reported absence of a significant correlation between bilirubin concentration and HDL-cholesterol. ${ }^{26}$

\section{Conclusion}

The level of serum bilirubin is negatively significantly associated with fasting blood glucose, cholesterol, LDL-cholesterol and 
triglycerides in type 2diabetic subjects while a non significant association of serum bilirubin was observed with BMI, WHR blood pressure $\mathrm{HbA1c}$ and HDL-chol.

\section{Acknowledgements}

This research work is the part of HEC Research Project "Molecular Mechanisms involved in the interactions of obesity, type 2diabetes mellitus and hypertension" (Ref \# 20-150/R and D/09(2890). I acknowledge the financial support of HEC to carry out my research.

\section{Conflicts of interest}

The authors declare that there are no conflicts of interest.

\section{Funding}

None.

\section{References}

1. Yeh SY, Doupis J, Rahangdale S, et al. Total serum bilirubin does not affect vascular reactivity in patients with diabetes. Vasc Med. 2009;14(2):129-136.

2. Adiels M, Olofsson SO, Taskinen MR, et al. Overproduction of very lowdensity lipoproteins is the hallmark of the dyslipidemia in the metabolic syndrome. J Arterioscler Thromb. Vasc Biol. 2008;28(7):1225-1236.

3. Taskinen MR. Type 2 diabetes as a lipid disorder. J Curr Mol Med. 2005;5(3):297-308.

4. Adiels M, Olofsson SO, Taskinen MR, et al. Diabetic dyslipidaemia. $J$ Curr Opin Lipidol. 2006;17(3):238-246.

5. Reasner CA. Reducing cardiovascular complications of type 2 diabetes by targeting multiple risk factors. J Cardiovasc Pharmacol. 2008;52(2):136-144.

6. Houten SM, Watanabe M, Auwerx J. Endocrine functions of bile acids. EMBO. 2006;25(7):1419-1425.

7. Bays HE, Goldberg RB. The 'forgotten' bile acid sequestrants: is now a good time to remember? Am J Ther. 2007;14(6):567-580.

8. Goldfine AB. Modulating LDL cholesterol and glucose in patients with type 2 diabetes mellitus: targeting the bile acid pathway. J Curr Opin Cardiol. 2008;23(5):502-511.

9. Tiribelli C, Ostrow JD. The molecular basis of bilirubin encephalopathy and toxicity: report of an EASL Single Topic Conference. J Hepatol. 2005;43(1):156-158.

10. Kapitulnik J. Bilirubin: an endogenous product of heme degradation with both cytotoxic and cytoprotective properties. J Mol Pharmacol. 2004;66(4):773-779.

11. Sedlak TW, Saleh M, Higginson DS, et al. Bilirubin and glutathione have complementary antioxidant and cytoprotective roles. Proc Natl Acad Sci. 2009;106(13):5171-5176
12. Vitek L, Jirsa M, Brodanova M, et al. Gilbert syndrome and ischemic heart disease: a protective effect of elevated bilirubin levels. Atherosclerosis. 2002;160(2):449-56.

13. Yesilova Z, Serdar M, Ercin CN, et al. Decreased oxidation susceptibility of plasma low density lipoproteins in patients with Gilbert's syndrome. J. Gastroenterol. Hepatol. 2008;23(10):1556-1560.

14. Chen YH, Chau LY, Chen JW, et al. Serum bilirubin and ferritin levels link heme oxygenase- 1 gene promoter polymorphism and susceptibility to coronary artery disease in diabetic patients. Diabetes Care. 2008;31(8):1615-1620.

15. Kawamura $\mathrm{K}$, Ishikawa $\mathrm{K}$, Wada $\mathrm{Y}$, et al. Bilirubin from heme oxygenase-1 attenuates vascular endothelial activation and dysfunction. Arterioscler Thromb Vasc Biol. 2005;25(1):155-160.

16. Rodella L, Lamon BD, Rezzani R, et al. Carbon monoxide and biliverdin prevent endothelial cell sloughing in rats with type I diabetes. Free Radic Biol Med. 2006;40(12):2198-2205.

17. Lewis GF, Carpentier A, Adeli K, et al. Disordered fat storage and mobilization in the pathogenesis of insulin resistance and type 2 diabetes. J Endocr Rev. 2002;23(2):201-229.

18. Bhuiyan AR, Srinivasan SR, Chen W, et al. Association of serum bilirubin with pulsatile arterial function in asymptomatic young adults: the Bogalusa Heart Study. Metabolism. 2008;57(5):612-616.

19. Lin JP, Vitek L, Schwertner HA. Serum bilirubin and genes controlling bilirubin concentrations as biomarkers for cardiovascular disease. Clin Chem. 2010;56(10):1535-1543.

20. Ndisang JF, Jadhav A. Heme oxygenase system enhances insulin sensitivity and glucose metabolism in streptozotocin-induced diabetes. Am J Physiol Endocrinol Metab. 2009;296(4):829-841.

21. Ndisang JF, Lane N, Jadhav A. The heme oxygenase system abates hyperglycemia in Zucker diabetic fatty rats by potentiating insulinsensitizing pathways. Endocrinology. 2009;150(5):2098-2108.

22. Ndisang JF, Lane N, Jadhav A. Upregulation of the heme oxygenase system ameliorates postprandial and fasting hyperglycemia in type 2 diabetes. Am J Physiol Endocrinol Metab. 2009;296(5):1029-1041.

23. Kharb S. Association of Serum Concentration of Total Bilirubin and Low Density Lipoprotein Cholesterol with Myocardial Infarction. World J Med Sci. 2006;1(2):93-94.

24. Lalitha B, Sairam Ch VS, Ushakiranmayi G, et al. Comparative study of oxidative stress, clinical complications and incidence of type-ii diabetics in different age groups. Int J Med Res. 2010;1:59-67.

25. Suryawanshi NP, Bhutey AK, Nagdeote AN, et al. Study of lipid peroxide and lipid profile in diabetes mellitus. Indian Journal of Clinical Biochemistry. 2006;21(1):126-130.

26. American Diabetes Association. Clinical Practice Recommendations. Position Statement: Diagnosis and Classification of Diabetes Mellitus. Diabetes Care. 2004;27:5-10. 